\title{
IMPLICATIONS OF ALTERNATIVE TREATMENT IN BREAST CANCER
}

Alane F. Lins', Geovana C. Silva', Guilherme de A. Rodrigues¹, Isabela C. Moreira', Lucas F. A. Mendonça1, Leticia L. C. Real'1, Luiza S. Coutinho²

${ }^{1}$ Centro Universitário de Anápolis - UniEVANGÉLICA - Anápolis (GO), Brazil.

2Pontifícia Universidade Católica do Goiás - Goiânia (GO), Brazil.

Objectives: The objective of this work is to correlate the development of breast cancer, alternative forms of treatment, and patient improvement. Methodology: It is based on a systematic review of 2018 and 2019 PubMed and Google Scholar databases. The keywords "Breast Neoplasms", “Complementary Therapies”, "Drug Therapy”, and "Plants, Medicinal” were used. Results: The unconventional therapies, more known as Complementary and Alternative Medicines (CAM), have become increasingly popular in patients with breast cancer. Fruit extract of graviola (IL-GFE), a metabolite called Annonaceous acetogenics (AAs) that has antiproliferative effect against breast cancer cell lines. The mechanism observed in MCF-7 cells (most recurrent adenocarcinoma among women) consists of an intrinsic apoptotic pathway. In addition to inhibit proliferation, its GFE also inhibits the metastasis process. On the other hand, the combined use of tamoxifen and bioactive compounds of Strobilanthes crispus showed strong inhibition to the cell lines MCF-7 and MDA-MB-231 to break the transmembrane potential of these cells and induce a marked apoptosis. Conclusion: From the data exposed, it is concluded that, currently, the combination therapy has become the hallmark of the breast cancer treatment because of the high incidence of tumor recurrence and progression of the disease after monotherapeutics treatments, including surgery, radiation therapy, hormonal therapy, and chemotherapy. Besides, the association of these alternatives present extra mechanisms on containment, eradication, and remission of the disease, with a greater effectiveness of treatment when compared to traditional monotherapy. 